\title{
Application Study of an Improved Chaos Genetic Algorithm
}

\section{ChaoGang Wang}

Faculty of Humanities, Yunnan College of Economic and Management, Kunming 650106, China

$$
\text { 39266097@qq.com }
$$

Keywords: chaos, genetic algorithm, application study.

\begin{abstract}
This paper presents an improved genetic algorithm based on chaotic characteristics, main combining with the rapid global convergence of genetic algorithm and optimization of chaotic sequence, through the singularity and the complexity of chaotic system to improve mutation operator of algorithm to obtain a better multi-objective optimal solution. At the same time, the application of the improved algorithm to the test process not only enriches the multi-objective optimization theory, but also provides an effective way to design an effective chaotic genetic algorithm.
\end{abstract}

\section{Chaotic Theory}

Chaotic Theory is scientific revolution [1] rising in these nearly thirty years, and it is listed as one of the greatest discovery with the relativity and quantum mechanics in twentieth Century. Quantum mechanics questions the physical causality in microscopic world, and chaos theory denies the decision causality including the Laplace type in macroscopic world. Chaos is a kind of non-periodic motions that's nonlinear system unique characteristic and widely exists, whose coverage relates to almost every branch of natural science and social science. Because the singularity and the complexity of chaotic system has not been completely understood, there is no fixed definition of chaos [2].

In 1975, Li Tianyan and York (J.A.Yorke) first made mathematical definition of chaos in the famous paper "Period implies chaos" in system [3].Li-Yorke theorem: given a continuous self-mapping $f: I \subset R$, if there is a set of uncountable $S \subset I$, it satisfies the following three conditions:

First, S doesn't contain periodic points.

Second, given any $X_{1}, X_{2} \in\left(X_{1} \neq X_{2}\right)$, there is $\limsup \left|f^{\prime}\left(X_{1}\right)-f^{\prime}\left(X_{2}\right)\right|>0$, $\liminf _{i \rightarrow \infty}\left|f^{\prime}\left(X_{1}\right)-f^{\prime}\left(X_{2}\right)\right|=0$. Here, $f^{\prime}()=.f(f(\ldots f())$.$) means recomposite function of t$.

Third, given any $X_{1} \in S$ and any periodic point of $f \quad P \in I$, then there is $\limsup _{i \rightarrow \infty}\left|f^{\prime}\left(X_{1}\right)-f^{\prime}(P)\right|>0$.

\section{Chaos genetic algorithm}

Chaotic evolutionary algorithm mainly uses the randomness and ergodicity of chaos. Use the method similar to the wave carrier, and map the parameters to be optimized into the interval of chaotic variables. Such as the use of logistic mapping, which take $\mu^{\mu}$ as 4 , and at this time to achieve complete chaos. By using chaos is sensitive to initial values and assign to the minor-differences initial values so can get i chaos characteristics.

$$
x(n+1)=4 x(n)(1-x(n))
$$

\section{Polynomial mutation}

Polynomial mutation is a mutation method proposed by Deb, and its variation form:

$$
v_{k}^{\prime}=v_{k}+\delta\left(u_{k}-l_{k}\right)
$$




$$
\delta=\left\{\begin{array}{c}
{\left[2 u+(1-2 u)\left(1-\delta_{1}\right)^{\eta_{m}+1}\right]^{\frac{1}{\eta_{m}+1}}-1, \quad \text { if } \quad r_{k}<0.5} \\
1-\left[2(1-u)+2(u-0.5)\left(1-\delta_{2}\right)^{\eta_{m}+1}\right]^{\frac{1}{\eta_{m}+1}} \text { if } \quad r_{k} \geq 0.5
\end{array}\right.
$$

In the formula $\delta_{1}=\left(v_{k}-l_{k}\right) /\left(u_{k}-l_{k}\right), \delta_{2}=\left(u_{k}-v_{k}\right) /\left(u_{k}-l_{k}\right)$, $u$ is a random number of an interval $[0,1], \eta_{m}$ is distribution index selected by a user and in the experiment $\eta_{m}$ is 11 .

\section{Chaotic mutation}

Expressions for the chaotic mutation: $c_{k}=p_{k}+\left(p_{k}^{u}-p_{k}^{l}\right) \delta_{k}$ Among them, $c_{k}$ is the offspring and $p_{k}$ is parent generation, $p_{k}^{u}$ is the upper bound of the parent component. $p_{k}^{l}$ is the lower bound. $\delta_{k}$ is a small step generated by chaotic sequences.

\section{The process of genetic algorithm [4]}

A) Initialization settings: set the modern evolution algebra counter $t=0$, the maximum evolution algebra $\mathrm{T}$, randomly generated $\mathrm{M}$ individuals as the initial population of $\mathrm{P}(0) \mathrm{B})$ Individual evaluation: Calculation of group $\mathrm{P}(\mathrm{T})$ in the fitness of each individual.C) Selecting operation: the selection operator acting on the group. The choice of the purpose is to direct individual genetic optimization to the next generation, or by paired crossover generates new individuals and then transmitted to the next generation. The choice of operation is based on individual fitness evaluation based on.D) Crossover calculation: crossover operator acting on the group. Is the crossover operator plays a central role in genetic algorithm.E) Mutation operation: make the mutation operator act on the group. That is changing the value for some loci of individuals in the population of the gene string.Group P (T) after the selection, crossover and mutation operator to be the next generation of group $\mathrm{P}(\mathrm{t}+1)$.F) Termination condition judgment: if $\mathrm{t}=\mathrm{T}$, then in the evolution process and the resulting with the maximum fitness of individuals as the optimal solution output, terminate the computation.

\section{The process of advance Chaos genetic algorithm}

(1)make sure the operation parameters: population size $\mathrm{M}$, the number of variables $\mathrm{L}$, crossover probability pc, mutation probability pm, the maximum number of iterations of N.(2) using chaos method in $[0,1]$ generate a list of initial values as the initial population.(3) using proportional selection operator to the current population to achieve the survival of the fittest.(4) mate the individuals randomly in a population of two-two, and generate a random number of each pair, and crossover probability decides whether to cross (arithmetic crossover).(5) take mutation operation on the current group, and the chaotic sequence is generated by the chaotic mapping here. First the number and the mutation probability of randomly generated determine whether to vary (non-uniform mutation).(6) accept the new individual by the probability, and each new individual generates a random number pc between 0 and 1 . If pc $<$ p, accept anew individual, unless, not.(7) calculate the fitness, then sort the population according to the fitness.(8) chaos mutation of individuals in a population. With the iterative approximation gradually to the superior solution, until two times before and after ones calculate the difference value of adaptation average value is less than the given small positive number.(9) if the maximum genetic algebra is reached, then end the operation and output the results, otherwise go to step3. 


\section{Comparison of commonly used algorithms of three kinds of common automatic test paper}

The three kinds of automatic generating test paper algorithm test is divided into two times, the first time for each type of test paper and test paper process test production time and accuracy, the experimental running time is $0-90$ seconds, the correct rate is 0 to $100 \%$. The following Tutu. For the second time in accordance with the relationship between the time and the number of papers papers three generating algorithm of production, the experimental running time is 0-80 seconds, test papers for 16072 sets $0-2000$. As shown in figure two.

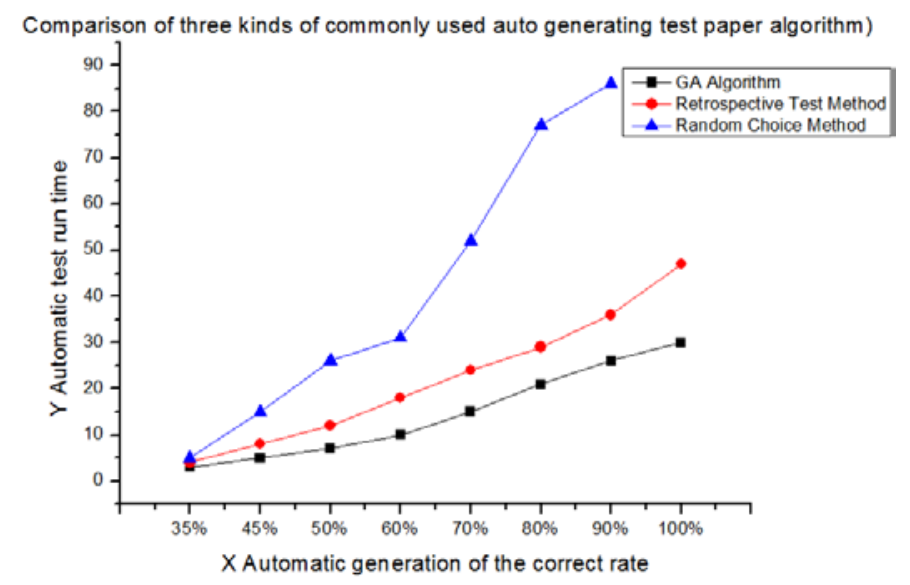

Fig. 1 Comparison of three algorithms loss time

From the above diagram, this heuristic genetic algorithm is usually used to generate solutions useful to optimization and search problems. Genetic algorithm fitness function selection improper circumstances might converge to local optimal [5], but cannot reach the global optimum. Genetic algorithm with adaptive global optimization and intelligent search technology, and the convergence at the auto generating test paper in the search process, the method compared to the other two methods, both are more optimal accuracy and time efficiency.

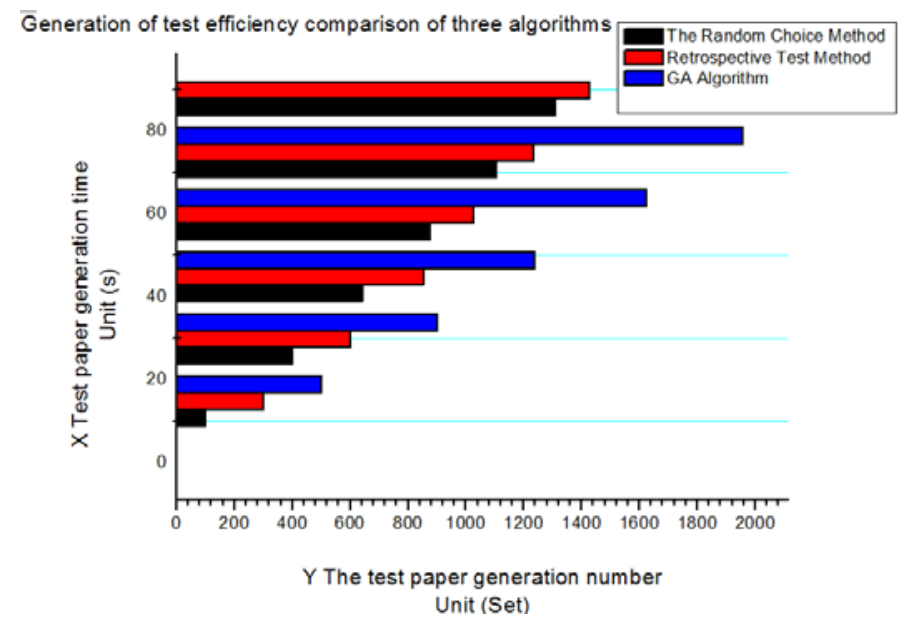

Fig. 2 The three algorithm accuracy rate

\section{Summary}

In the genetic algorithm, the mutation operator is a very important part of the evolutionary algorithm, and is paid more attention by researchers in the single objective evolutionary algorithm. This paper studies the chaotic mutation operator in a multi-objective evolutionary algorithm. As the basis of chaos optimization is optimized through the mutation operator, this paper focuses on the improvement of mutation operator. Because the hybrid chaotic search strategy integrates the characteristic of chaotic search and other optimization mechanism of mutual learning, the 
convergence speed and the efficiency of the general is better than the simple chaotic search method. Therefore, the hybrid chaotic search algorithm of chaos search and other optimization combination mechanism is an important research direction of chaos optimization theory and its application.

\section{References}

[1] Wu Xiangxing, Chen Zhong et al. Chaos theory introduction [M] Shanghai science and Technology Literature Press.p.26-27.

[2] Bruce Schneier . Wu Shizhong, Zhu Shixiong, Zhang Wenzheng et al. translation. Application of Cryptography - Protocol algorithms and C source program in Second Edition [M]. Beijing Mechanical Industry Express1996.

[3] T.Y.Li,J.A.YOrke.Period three implies chaos[J].Amer.Math.Monthly,1975,(82)p.985-992.

[4] Ding Xue, A Study on the Image Classification Techniques Based on Wavelet Artificial Neural Network Algorithm, Advanced Manufacturing and Information Engineering, Intelligent Instrumentation and Industry Development.

[5] Ding Xue. A study of evolutionary algorithms based on muti-objective pareto optimality, Journal of Chemical and Pharmaceutical Research,2014.6(7) p.2547-2553. 